\title{
Fuzzy PID Control System In Industrial Environment
}

\author{
Hong $\mathrm{HE}^{1, \mathrm{a}^{*}}$, Yu LI ${ }^{1}$, Zhi-Hong ZHANG ${ }^{1,2}$, Xiaojun XU1 \\ ${ }^{1}$ Tianjin Key Laboratory for Control Theory and Application in Complicated Systems, \\ Tianjin University of Technology, china \\ ${ }^{2}$ Tianjin Broadcast Television Development Ltd, Tianjin, 300381, China \\ aheho604300@126.com
}

Keywords: Industry; Pneumatic; Fuzzy PID control; Simulation

\begin{abstract}
To solve the control problem of industrial pneumatic actuate, this paper makes an in-depth research on fuzzy control theory and PID control theory to propose a fuzzy PID pneumatic control strategy based on the parameter self-tuning and designs a fuzzy PID controller.It makes a simulation analysis on the pressure control and simulation results show that: the fuzzy PID pneumatic control systems in industrial environment ensures the controlled object with good dynamic and static quality a good prospect for engineering application.
\end{abstract}

\section{Introduction}

Because of the growing demand, automation and intelligent play an increasingly important role in our daily life. Especially for the industrial pneumatic control systems in today's complex industrial environment. Based on the advantages and limitations of fuzzy control and PID control, this paper combines the fuzzy control with PID control to form the fuzzy PID control, which can achieve better control effect and improve the control precision of the pneumatic system, so as to control the industrial pneumatic systems precisely.

\section{Principle Of Fuzzy Control And PID Control Theory}

\section{Fuzzy control theory}

With the complication of automatic control system's controlled objects, the control system has the characteristics of multi-input, multi-output of strong coupling, parameter variability and nonlinear $^{[1]}$.

Structure of the fuzzy controller in Figure.1. As can be seen from the figure below, the fuzzy controller is composed of fuzzy, knowledge base, fuzzy inference, and ambiguity.

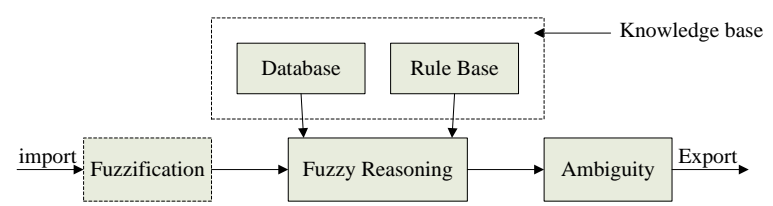

Figure.1 Fuzzy controller structure diagram

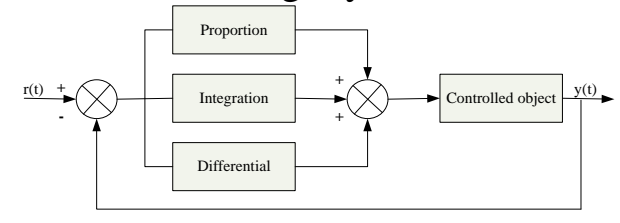

Figure.2 PID controller schematics

\section{PID control theory}

PID control's characteristic is only adjust the controller parameters, i.e., the proportional coefficient $K_{P}$, the integral coefficient $K_{I}$ and the differential coefficient $K_{D}$, which can obtain satisfactory results. PID controller block diagram is shown in Figure.2.

According to the control deviation $e(t)$ formed by the set target value $r(t)$ and the actual output value $y(t)$, the paper combines this deviation proportion, integral and differential to form the control amount via a linear combination, controlling the controlled object .

Controller equation 
$e(t)=r(t)-y(t)$

The controller output equation $u(t)$ for the time domain

$$
u(t)=K_{P} e(t)+K_{I} \int e(t) d t+K_{D} \frac{d e(t)}{d t}
$$

From the formula(2), the adjustment only affects the corresponding coefficients variation in the formula.

\section{Fuzzy PID Control System}

Based on the control principle, the fuzzy PID control system design makes a fuzzy linguistic variables selection to determine the membership function and the development of fuzzy rules, ultimately realizing defuzzification ${ }^{[2]}$.

\section{Fuzzy PID control theory}

PID controller block diagram in Figure. 3 are shown.

The nature of Parameter self-tuning fuzzy PID control is based on the conventional PID control, taking the error $e^{e}$ of control target value and the error's change ratio ${ }^{e_{c}}$ as input, so that the controlled object has a good dynamic and static performance indicators.

\section{Determine fuzzy linguistic variables}

The system error $e$ and error rate $e_{c}$ of change, $K_{P}, K_{I}, K_{D}$ as input linguistic variables fuzzy controller,for the output linguistic variables. Their range defined as the basic domain on fuzzy sets:

$$
e, e_{c}, K_{P}, K_{I}, K_{D}=(-4,-3,-2,-1,0,1,2,3,4)
$$

Its fuzzy subset $e, e_{c}=\{\mathrm{NB}, \mathrm{NS}, \mathrm{ZE}, \mathrm{PS}, \mathrm{PB}\}$, Subset of elements representing the negative big, negative small, zero, positive small, $\mathrm{CP}$. This division general requirements for quality control applications.

The basic theory of continuous change within the gamut discrete amount of grading and obfuscate. Components of the velocity deviation $e$ and the variation $e_{c}$ range of the second set of $[4,4]$, if not in this range, by the linear transformation formula (3) will be the value in the [a, b] to the continuous transition between the amount of between [-4 4].

$$
y=\frac{8}{b-a}\left(x-\frac{a+b}{2}\right)
$$

\section{Determine the membership function}

Here selecting the triangular as the membership functions for linguistic variables, shown in Figure.4.

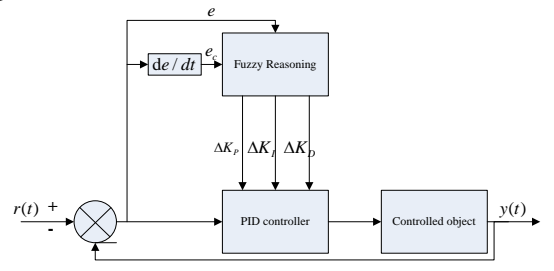

Figure.3 Fuzzy PID controller structure diagram

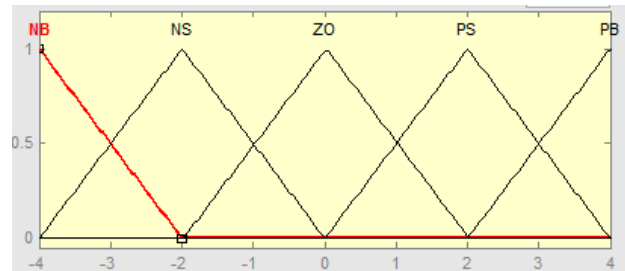

Figure.4 Membership function diagram

In fact, the membership function's shapes (triangles, trapezoids, or normal distribution, etc.) don't affect the control effect greatly. Therefore, $e$ and $e_{c}$ used the same membership function and $K_{P}, K_{I}, K_{D}$ also uses the same membership function. Which can determine $e$ and $e_{c}$, and $K_{P}, K_{I}, K_{D}$ membership assignment table ${ }^{[3]}$.

\section{Fuzzy rules}

Using fuzzy rules to adjust the PID parameters, we should have a deep understanding of the impact of the PID's three parameters $K_{P}, K_{I}, K_{D}$ on the static and dynamic characteristics of 
control system, when the system error $e$ and error's change ratio $e_{c}$ are not the same, we will make reasonable adjustments in PID parameters to make the system tends to the target value fast and maintain stable.

Through the analysis of the system step response curve and considering the impact of the three parameters $K_{P}, K_{I}, K_{D}$ of PID controller on the system performance, the principles of fuzzy PID tuning parameters are summarized as follows:

1.When the process value and the set value difference is large, no matter how $e_{c}$ changes, we should control proportion strongly to reduce the absolute value of the error, in order to avoid the integral saturation, we can select a larger $K_{P}$, which is generally zero, we select a smaller $K_{D}$ for reducing interference, ${ }^{[4]}$.

2. When $e$ and $e_{c}$ are the same number, it's indicated that the error changes in the direction of increasing absolute value, if the absolute value $e$ is larger, we should control strongly and decrease $e$ rapidly, at this time we can select a larger $K_{P}$, a smaller $K_{I}$ and a medium $K_{D}$ to improve the dynamic and steady-state performance; if the absolute value $e$ is small, we can control generally to change the variation trend of error, we can select a medium $K_{P}$, while select a larger $K_{I}$ and a smaller $K_{D}$ to improve the steady-state performance of the system.

3. $e$ with the opposite sign indicates that the error changes in the direction of reduced absolute value. If the absolute value of $e$ is large, we should control generally and quickly reduce the error's absolute value, at this time we can select a medium $K_{P}$, a smaller $K_{I}$ and a medium $K_{D}$ to improve the dynamic performance and steady-state performance; if $e$ 's absolute value is small, we can select a smaller $K_{P}$, a larger $K_{I}$ and a smaller $K_{D}$ to improve the steady-state performance of the system and avoid oscillations.

Through the above analysis, we respectively establish a fuzzy control rule table

Table $1, K_{P}, K_{I}, K_{D}$ the fuzzy inference rules

\begin{tabular}{|l|l|l|l|l|l|}
\hline$e_{c}$ & NB & NS & ZO & PS & PB \\
\hline NB & PB NB PS & PB NB ZO & PB NB ZO & PS NB ZO & ZO ZO PS \\
\hline NS & PB NB NB & PS NS NB & PS NS NS & ZO ZO ZO & NB ZO PS \\
\hline ZO & PS NS NB & PS NS NB & ZO ZO NS & NS PS ZO & NB PS PS \\
\hline PS & PS NS NB & ZO ZO NS & NS PS NS & NS PS ZO & NB PB PS \\
\hline PB & ZO ZO PS & NS PS ZO & NS PS ZO & NB PB ZO & NB PB PS \\
\hline
\end{tabular}

\section{Defuzzification}

Considering the characteristics of the system and the simplify of the calculation process, this paper clarifies through the largest membership process. Here taking the $\Delta K_{P}$ and the first fuzzy rule for example, according to the first row of the reasoning table of $\Delta K_{P}$ fuzzy control rules, we can obtain the fuzzy set (express in Zadeh representation), i.e.

$$
\Delta K_{P 1}=\frac{0.0}{-4}+\frac{0.0}{-3}+\frac{0.0}{-2}+\frac{0.0}{-1}+\frac{0.0}{0}+\frac{0.0}{1}+\frac{0.0}{2}+\frac{0.0}{3}+\frac{0.0}{4}
$$

Seen from the fuzzy set, the value of $\Delta K_{P}$, which corresponds to the maximum value of membership, is four, and the fuzzy control rules of corresponding $e$ (error) and $e_{c}$ (error's rate of change) are NB (negative big), the values in the corresponding domain are -4. By that analogy, we can obtain the fuzzy adjustment set of the remaining parameters, completing the anti-fuzzy of the fuzzy reasoning. 


\section{Fuzzy PID Control Simulation With Interference And Result Analysis}

PID control and fuzzy PID control simulation model with interference

By deforming the equation of the pneumatic valves switches in the control system of pneumatic climbing wall trolley into PID control equation, we can obtain that for open-loop transfer function is the controlled object.The simulation model shown in Figure.5. The step1 and step 3 are the same added step disturbance signal .

\subsection{Analyze the results of simulation with interference}

Under the stable operating conditions, which the output pressure is $0.35 \mathrm{MPa}$, the system suddenly add a $10 \mathrm{~L} / \mathrm{min}$ carrying capacity step signal at $2.35 \mathrm{~s}$. The response of the two algorithms (one without interference one with interference) is shown in Figure.6.

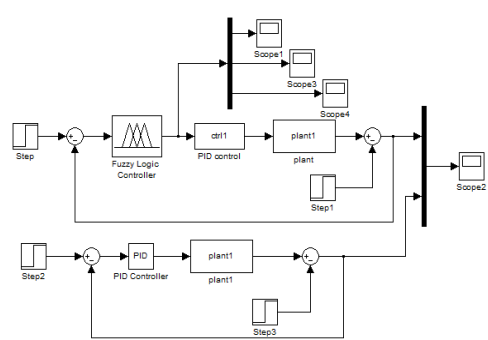

Figure.5 Interfere with PID control and fuzzy PID control simulation model

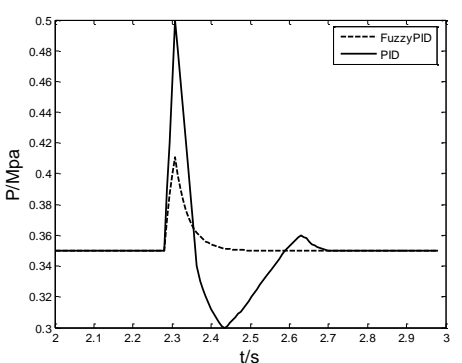

Figure.6 Fuzzy PID control and PID control air pressure changes in the interference graph

Table 2. Comparison of the two control algorithms

\begin{tabular}{|l|c|c|}
\hline & Traditional PID control algorithm & $\begin{array}{c}\text { Parameter self-tuning fuzzy PID } \\
\text { control algorithm }\end{array}$ \\
\hline Overshoot $/ \mathrm{Pa}$ & 1.5 & 0.05 \\
\hline Adjustment time /S & 0.4 & 0.1 \\
\hline
\end{tabular}

The comparison of the two algorithms' response are shown in Table 2, it is clear that the overshoot of the traditional PID control algorithm is larger and the pressure adjustment is longer; Parameters self-tuning fuzzy PID control algorithm is able to achieve fast response of pressure, and has no overshoot, effectively increasing the steady-state control accuracy of the pressure.

\section{Conclusion}

This paper focuses on the lag and time-varying characteristics of pneumatic system, and combines the fuzzy control and adaptive PID control to design a fuzzy adaptive PID control system. We calculate the parameters $\Delta K_{P}, \Delta K_{I}, \Delta K_{D}$ of a complete blur adjustment table by the fuzzy reasoning, realizing the online self-tuning of PID parameters to meet the accuracy requirements of pneumatic control system under the complex industrial environment; and establish a fuzzy PID simulation model, the simulation results show that compared to PID control, the fuzzy PID control can achieve stability in a relatively short time and has small overshoot; and has a strong adaptive ability to better adapt to the situation of sudden external disturbance into the system during the control process.

\section{References}

[1]Shi Dequan, Gao Guili, Gao Zhiwei, Xiao Peng. Application of Expert Fuzzy PID Method for Temperature Control of Heating Furnace[J].ProcediaEngineering,2012,29.

[2] Li Qingchun, Chen Deyao Kind of PID fuzzy controller (fuzzy + PID-type) [J] Control and Decision, 2009,07: 1038-1042.

[3] Zhang Enqin, Shi Songjiao, Weng are a new class of novel fuzzy control method based on PID control [J] Shanghai Jiaotong University, 2000. 
[4] Chen Xuesong learning and its application [D] in the robot system Strengthen. Guangdong University of Technology, 2011. 